\section{Dynamic expression and essential functions of Hes 7 in somite segmentation}

\author{
Yasumasa Bessho, ${ }^{1}$ Ryoichi Sakata, ${ }^{1}$ \\ Suguru Komatsu, ${ }^{2}$ Kohei Shiota, ${ }^{2}$ \\ Shuichi Yamada, ${ }^{1}$ and Ryoichiro Kageyama ${ }^{1,3}$ \\ ${ }^{1}$ Institute for Virus Research, Kyoto University, Kyoto \\ 606-8507, Japan; ${ }^{2}$ Department of Anatomy and \\ Developmental Biology, Kyoto University Graduate School \\ of Medicine, Kyoto 606-8501, Japan
}

The basic helix-loop-helix (bHLH) gene Hes7, a putative Notch effector, encodes a transcriptional repressor. Here, we found that Hes 7 expression oscillates in 2-h cycles in the presomitic mesoderm (PSM). In Hes7-null mice, somites are not properly segmented and their anteriorposterior polarity is disrupted. As a result, the somite derivatives such as vertebrae and ribs are severely disorganized. Although expression of Notch and its ligands is not affected significantly, the oscillator and Notch modulator lunatic fringe is expressed continuously throughout the mutant PSM. These results indicate that Hes 7 controls the cyclic expression of lunatic fringe and is essential for coordinated somite segmentation.

Received July 24, 2001; revised version accepted August 23, 2001.

Somites, the segmental units for the metameric structures such as skeletal muscles, vertebrae, and ribs are sequentially generated in a head-to-tail order by segmentation of the mesenchymal tissue called the presomitic mesoderm (PSM). The segmentation occurs periodically at the anterior end of the PSM, and this periodic segmentation has been suggested to be regulated by a molecular clock (Cooke 1998; Dale and Pourquié 2000). The first evidence for the molecular clock for somite segmentation was provided by a report about the chick basic helix-loop-helix (bHLH) gene chairy1 (Palmeirim et al. 1997). chairy1 expression initiates as a broad band in the posterior PSM, moves anteriorly, and reaches the anterior end of the PSM as a narrow band, which corresponds to the posterior part of a newly formed somite (Palmeirim et al. 1997). This sequence of chairy1 expression is repeated, and each cycle is correlated with formation of one somite. Similar dynamic expression is also observed for other chairy1-related bHLH genes, such as zebrafish Her1 and mouse Hes1 (Holley et al. 2000; Jouve et al. 2000; Sawada et al. 2000). Interestingly, the dynamic chairy1 expression is not the result of cell movement but attributable to wave-like propagation through the PSM

[Key Words: bHLH; Hes7; lunatic fringe; Notch; somite segmentation; oscillation]

${ }^{3}$ Corresponding author.

E-MAIL rkageyam@virus.kyoto-u.ac.jp; FAX 81-75-751-4807.

Article and publication are at http://www.genesdev.org/cgi/doi/10.1101/ gad.930601. cells (Palmeirim et al. 1997). For the wave-like propagation, chairy1 expression should oscillate synchronously in the PSM, but the exact mechanism for the synchronous oscillation remains to be determined.

Recent studies suggest that Notch signaling regulates the synchronously oscillating gene expression in the PSM and coordinated segmentation of somites. In zebrafish, mutation for the Notch pathway is likely to desynchronize the oscillating gene expression (a random mixture of PSM cells that express different levels of gene expression) and this desynchronization may lead to a loss of the coordinated somite segmentation (Holley et al. 2000; Jiang et al. 2000). Similarly, in mice, Notch signaling is essential for oscillating gene expression (Jouve et al. 2000) and coordinated segmentation of somites: mutations for Notch1, the ligands Delta-like 1 (D111) and D113, and other regulators such as Presenilin, $R B P-I$, and lunatic fringe (Lfng) all exhibit defects of somite segmentation (Swiatek et al. 1994; Conlon et al. 1995; Oka et al. 1995; Hrabe de Angelis et al. 1997; Wong et al. 1997; Evrard et al. 1998; Kusumi et al. 1998; Zhang and Gridley 1998; del Barco Barrantes et al. 1999; Donoviel et al. 1999; Huppert et al. 2000; Koizumi et al. 2001). Among these molecules, Lfng expression also oscillates in the PSM like chairy1-related genes (Forsberg et al. 1998; McGrew et al. 1998; Aulehla and Johnson 1999|, whereas expression of most other Notch pathway genes does not, suggesting that Lfng and chairy1-related genes are the key regulators to convert the temporal component (oscillation) to the spatial component (segmentation). Unlike $L f n g$, however, the significance of chairy1-related genes in somite segmentation is still obscure because the Hes1 mutation does not affect somitogenesis in mice (Ishibashi et al. 1995; Jouve et al. 2000) and other species lacking chairy1-related genes are not available.

Recently, we isolated a new chairy1-related gene from mouse, named Hes 7 (Bessho et al. 2001). Hes 7 encodes a Hes-like transcriptional repressor and its expression is controlled by Notch signaling. Interestingly, Hes 7 is expressed in the PSM in a dynamic manner (Bessho et al. 2001), raising the possibility that Hes7 expression is cyclic in the PSM. Here, we found that Hes7 expression oscillates in 2-h cycles in the PSM synchronously with Lfng expression. Strikingly, in mice mutant for Hes7, somites are not properly segmented and their anteriorposterior $(\mathrm{A}-\mathrm{P})$ polarity is disrupted. As a result, the somite derivatives, such as vertebrae and ribs, are severely disorganized. Importantly, the oscillator Lfng is expressed continuously throughout the mutant PSM and therefore the oscillating expression is disrupted. These results indicate that $\mathrm{Hes} 7$ controls the cyclic expression of $L f n g$ and is essential for somite segmentation.

\section{Results and Discussion}

\section{Dynamic expression of Hes7 in the PSM}

Hes7 displays various expression patterns in the PSM even at the same developmental stages (Fig. 1A), raising the possibility that Hes 7 expression oscillates in the PSM. To investigate this possibility, we compared the expression pattern of Hes7 and the oscillator Lfng in the same embryos. The caudal parts of embryonic day (E)9.5 


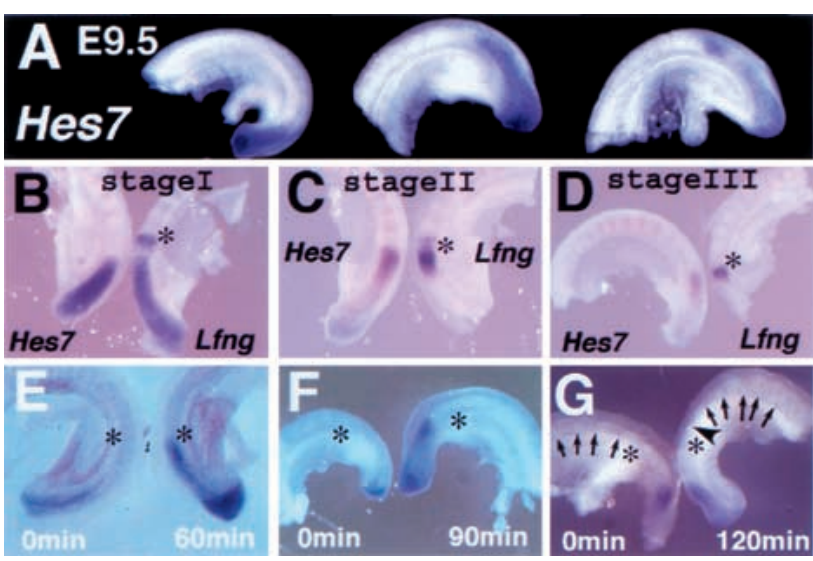

Figure 1. Dynamic expression of Hes7 in the PSM. (A) Variable expression patterns for Hes7 of E9.5 embryos. $(B-D)$ Comparison of the expression of Hes7 (left) and Lfng (right) in the bisected caudal portions of E9.5 embryos. The expression patterns of the two genes were variable, but similar to each other in all three stages (stage I, $n=4$; stage II, $n=4$; stage III, $n=2$ ). Each pair is dissected from the same embryo. The established somites were stained with the Uncx4.1 probe for spatial alignment. (EG) Hes7 is differentially expressed after explant culture. The right halves were cultured for $60 \mathrm{~min}(E), 90 \mathrm{~min}(F)$, or $120 \mathrm{~min}$ $(G)$, and compared with the left halves, which were fixed immediately. $(G)$ Somite boundaries and a newly formed boundary are indicated by arrows and arrowhead, respectively. Somite -I is indicated by asterisks.

mouse embryos were cut into halves along the midline and the two halves were subjected separately to in situ hybridization with Hes 7 and Lfng probes (Fig. 1B-D). It was reported previously that oscillating gene expression pattern is categorized into three distinct stages (Palmeirim et al. 1997). During stage I, a broad expression domain appears in the posterior PSM; during stage II, the expression moves anteriorly and becomes narrower; and during stage III, the expression is present in somite -II (somite -I is the next somite to form and somite -II is the one to form after somite -I). During stage I, a broad Hes 7 expression domain appeared in the posterior PSM like Lfng, although Lfng expression still remained in somite -II (Fig. 1B). During stage II, Hes 7 expression moved anteriorly (Fig. 1C) and, during stage III, Hes7 was expressed in somite -II like Lfng, but Hes 7 expression was extended caudally compared with $L f n g$ expression (Fig. 1D). These results indicate that, although Lfng expression persists in presumptive somites for a longer period, the main expression domain of Hes 7 and Lfng in the PSM is well overlapped, suggesting that expression of these two genes oscillates in the same phases.

To clarify the oscillating expression of Hes7 further, we carried out explant culture experiments. The posterior part of E9.5 mouse embryos was cut along the midline and one half piece was fixed immediately and the other half was cultured for various time before fixation. Both pieces were then hybridized with a Hes 7 probe (Fig. $1 E-G)$. When one half was cultured for 60 or $90 \mathrm{~min}$, the expression patterns of Hes7 were different from those of the uncultured half (Fig. 1E,F). In contrast, when cultured for $2 \mathrm{~h}$, the explants had a new somite, but the Hes 7 expression pattern became very similar to the uncultured pattern (Fig. 1G), indicating that Hes7 expres- sion oscillates in 2-h cycles. Taken together, these results indicate that Hes7 expression oscillates synchronously with Lfng expression in the PSM. In addition, each cycle seems to be linked with generation of one somite.

In chick, the oscillation of chairy1 expression does not require de novo protein synthesis, whereas that of $L f n g$ does (Palmeirim et al. 1997; McGrew et al. 1998). We next examined whether cyclic expression of Hes 7 requires de novo protein synthesis by culturing the caudal parts of mouse embryos in the presence of cycloheximide. Cycloheximide treatment blocked cyclic expression of Hes7 (data not shown), suggesting that Hes7 expression depends on de novo protein synthesis. This result contrasts with that of chairy1, but the molecular mechanism for this difference between Hes 7 and chairy 1 remains to be determined.

\section{Generation of Hes7-mutant mice}

To understand the role of Hes 7 in somitogenesis, the Hes 7 gene was disrupted by homologous recombination in embryonic stem (ES) cells. Most of the coding region of Hes 7 was removed and, instead, lacZ gene was placed under the control of the Hes7 promoter (Fig. 2A). Chimeric mice were generated from the mutant ES cells and bred to produce heterozygous mutant mice. Hes 7 heterozygous mutant mice looked normal overall. Forty-three percent of them (51 out of 118), however, had kinked tails (Fig. 2C), suggesting that the dose of Hes7 gene is important for normal tail structure. Intercrossing of the

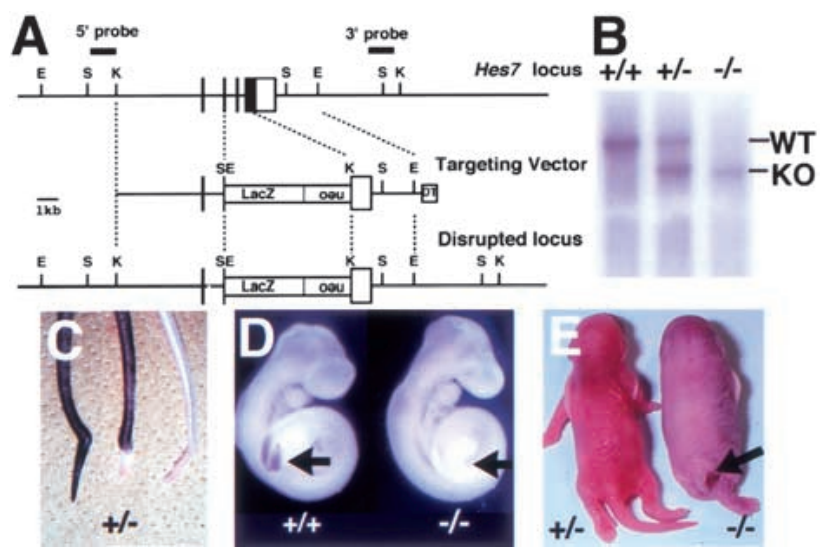

Figure 2. Generation of Hes7-null mice. (A) Targeting strategy. The top line shows the structure of the wild-type Hes 7 gene and the middle line shows the structure of the targeting vector. Most of the coding region of Hes7 was replaced by IRES-LacZ and PGK-neo (inverted orientation). The bottom line indicates the resultant disrupted locus. Diphtheria toxin gene (DT) was used for negative selection. (E) EcoRV; (S) SacI; (K) KpnI. The positions of 5 '-external and 3 '-external probes are indicated on top. (B) Southern blot analysis. The $5^{\prime}$-external probe detected 10-kb wild-type and 7-kb mutant bands of SacI-digested genomic DNA. $(C)$ Kinked tails of adult heterozygous mutants. $(D)$ Whole-mount in situ hybridization for Hes 7 of wild-type (left) and homozygous-mutant (right) embryos at E9.5. In the mutant embryo, Hes 7 expression is completely missing (arrows). (E) The appearance of heterozygous- (left) and homozygous-mutant (right) neonates. A homozygous mutant has a short trunk and a short tail (arrow). 
heterozygous mice generated Hes7 homozygous mutant mice (Fig. 2B), which lacked Hes7 expression (Fig. 2D, arrows). Hes7-deficient neonates had short trunks and tails, but their head and limbs appeared grossly normal (Fig. 2E). Most of them died within a few hours after birth, apparently from respiratory failure. The lung did not expand in Hes7-deficient mice, probably because of abnormality of vertebrae and ribs (see Fig. 3F). A few homozygous mutants, however, survived to adulthood.

\section{Segmentation defects of Hes7-deficient mice}

Because the external appearance of Hes7 homozygous neonates suggested malformations of skeletons, we next made skeletal preparations of neonates (Fig. 3A-F). No abnormality was observed in the skeletons of heterozygous mutant mice except for the tail (Fig. 3A,C,E). In contrast, in homozygous neonates, severe segmentation defects of axial skeletons were observed (Fig. 3B). The vertebrae and ribs were abnormally formed (Fig. 3D,F).

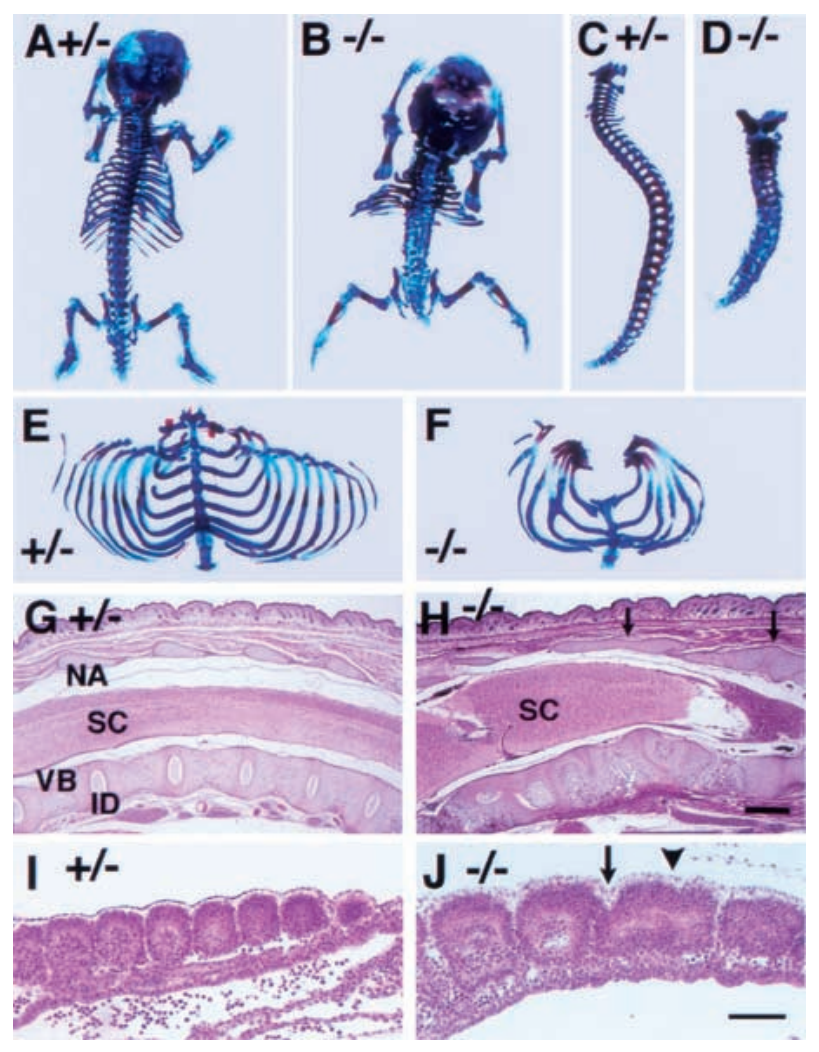

Figure 3. Segmentation defects in Hes7-null mice. $(A-F)$ Neonates of heterozygous $(A, C, E)$ and homozygous $(B, D, F)$ mutants were stained for bone (red) and cartilage (blue). $(A, B)$ Dorsal view of the whole neonates; $(C, D)$ lateral view of vertebrae and; $(E, F)$ dissected ribs and sternums. $(G, H)$ HE staining of sagittal sections of Hes7(+/-) $(G)$ and Hes7(-/-) (H) neonates. In Hes7(-/-) embryos, vertebral bodies and neural arches (arrows) are fused, and the patterning of vertebral bodies and intervertebral disks is defective. (ID) Intervertebral disk; (NA) neural arch; (SC) spinal cord; (VB) vertebral body. (I,J) Parasagittal sections of E9.5 embryos. Compared with Hes $7(+/-)(I)$, somites are irregularly arranged in Hes7(-/-) embryos $(J)$. Incomplete and almost complete fusion of epithelial somites are indicated by arrow and arrowhead, respectively. Bar, $1 \mathrm{~mm}(G, H) ; 100 \mu \mathrm{m}(I, T)$.
Vertebral bodies and neural arches were fused to each other throughout the entire length of the vertebral column, and the total length of vertebrae was significantly short (Fig. 3C,D). The ribs were fused and bifurcated, and the symmetry of right-left axis was lost. The number of rib pairs was reduced to eight to nine (normally 13), and the thoracic space was severely reduced (Fig. 3E,F). Sagittal sections of neonates also showed segmentation defects of axial skeletons in homozygous mutants (Fig. $3 \mathrm{G}, \mathrm{H})$, such as malformation and fusion of vertebral bodies, intervertebral disks, and neural arches (Fig. 3H). There was no difference in ossification among neonates of the three genotypes (data not shown), however, indicating that segmentation was primarily affected in Hes 7 deficient mice.

To investigate the abnormal segmentation further, we examined the somite formation of Hes7-mutant embryos. In wild-type and heterozygous embryos, a constant size of epithelial somites were formed repeatedly (Fig. 3I), whereas in homozygous embryos, they were irregular in both size and shape and the boundaries between somites were less clear (Fig. 3J, arrow and arrowhead). In addition, the left-right symmetric somite formation was disrupted in the homozygous embryos /data not shown). These results indicate that, in Hes7-deficient mice, somite segmentation occurred uncoordinatedly, and it is likely that this abnormal segmentation leads to severe defects of axial skeletons.

\section{Defects of somite patterning in Hes7-deficient embryos}

To examine the defects of somite segmentation further, we next analyzed several PSM-expressing genes. Because lac $Z$ was placed under the control of the Hes 7 promoter in the mutant allele, we first performed X-gal staining to determine whether the Hes 7 promoter activity was affected in Hes7-null mice. In Hes7(+/-) embryos, X-gal staining was observed in several caudal somites as well as in the PSM, probably because the lacZ product is stable (Fig. 4A). The staining was not restricted to the anterior or posterior half but observed in the whole region of each somite (Fig. 4A, lower left panel). In a Hes 7(-/-) embryo, staining in caudal somites was fused and the boundary between somites I and -I was not clear (Fig. 4A, right panels). Interestingly, in the Hes7(-/-) embryos, the intensity of X-gal staining was much stronger than in the Hes7(+/-) embryos (Fig. 4A), which cannot be explained by two copies of the lacZ gene, suggesting that the Hes 7 promoter activity is up-regulated in the absence of Hes7. Because Hes 7 is a transcriptional repressor (Bessho et al 2001), these data suggest that Hes 7 may repress its own expression.

The bHLH gene Paraxis is essential for formation of epithelial somites (Burgess et al. 1996). Paraxis expression was not changed significantly in the PSM of Hes7null embryos, although the expression in the mutant somites was fused (Fig. 4B). This observation is consistent with the above results in that, although irregular, epithelial somites were formed in Hes 7 homozygous embryos (Fig. 3J). Another bHLH gene, pMesogenin, regulates expression of Delta/Notch pathway molecules and controls somitogenesis (Yoon and Wold 2000). pMesogenin expression was not changed significantly in the PSM of Hes7-null embryos (Fig. 4C). Expression of the bHLH gene Mesp2, which specifies the anterior half of each somite (Saga et al. 1997), was reduced slightly com- 


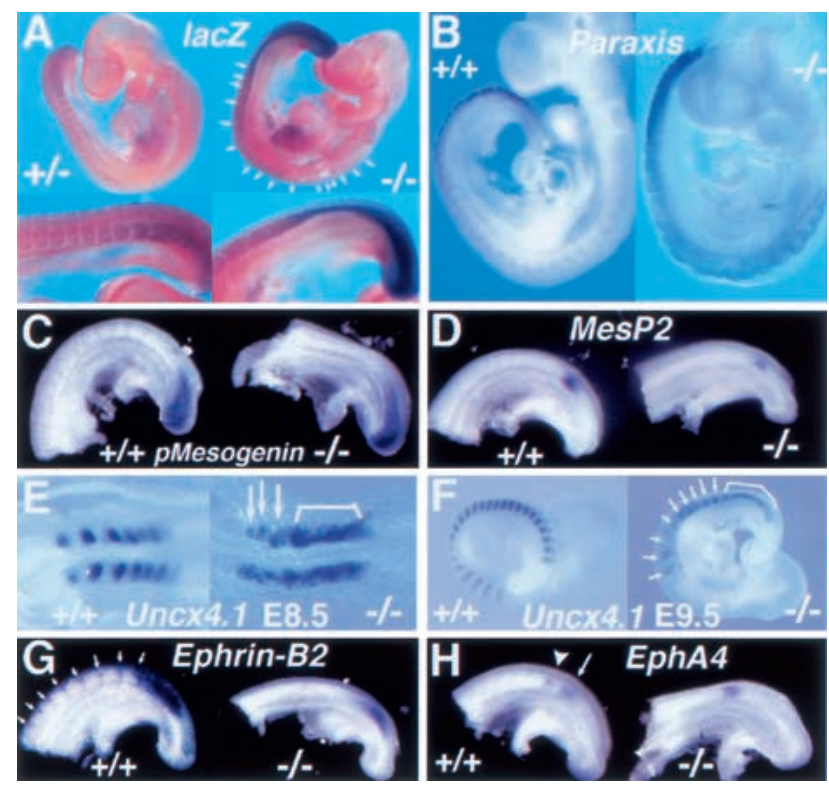

Figure 4. Defects of somitogenesis in Hes7-null embryos. $(A)$ Whole-mount lacZ staining. In a Hes $7(+/-)$ embryo, weak staining is observed in the PSM and several caudal somites. In contrast, in a Hes $7(-/-)$ embryo, much stronger staining is observed in the PSM and irregular somites. A higher magnification is shown in the lower panels. $(B-H)$ Expression of Paraxis $(B), p M e$ sogenin $(C)$, MesP2 (D), Uncx4.1 (E,F), Ephrin-B2 (G), and EphA4 $(H)$ was examined by in situ hybridization. $(E, F)$ The disorder of Uncx 4.1 expression is more severe in the caudal parts (brackets) than in the rostral parts (arrows). In $E$, rostral is to the left. $(G)$ Ephrin-B2 expression disappears in somites but remains in the PSM of Hes7-null embryos. $(H)$ In the wild type, EphA4 is expressed in the anterior half of somite I (arrowhead) and the PSM (arrow). In Hes7-null embryos, the expression in somite I disappears.

pared with the wild type but remained in the anterior PSM of Hes7-null embryos, as in the wild type (Fig. 4D). Therefore, Hes 7 mutation did not significantly affect expression of the PSM genes, except for its own promoter activity.

To determine the A-P polarity of formed somites, we next examined the anterior-half- or posterior-half-specific markers. The homeobox gene Uncx4.1, which controls sclerotome development (Mansouri et al. 2000), was expressed in the posterior half of each somite of the wild type (Fig. 4E,F). In contrast, in Hes7-null mice, the expression was severely disorganized (Fig. 4E,F). Interestingly, the disruption of Uncx4.1 expression was milder in the rostral part (Fig. 4E,F, arrows) than in the caudal part (Fig. 4E,F, brackets), which contained newly formed somites. At E8.5, the first four Uncx 4.1 $1^{+}$somites were irregularly separated (Fig. 4E, arrows), whereas the posterior somites were fused in Hes7-null embryos (Fig. 4E, bracket). In contrast, at E9.5, the first 10 somites were separated and the Uncx4.1 expression was restricted to the posterior half of each somite in Hes7-null embryos (Fig. 4F, arrows), whereas the caudal somites were fused (Fig. 4F, bracket). Therefore, it is likely that the initial segmentation and A-P patterning defects of somites are partially corrected at later stages in Hes7-null embryos. This correction could be involved in sorting of the ante- rior-half and posterior-half somite cells because they are known to have different adhesive properties (Stern and Keynes 1987). Expression of another posterior marker, Ephrin-B2, became very weak and almost undetectable in the somites of Hes7-null mice, whereas it was observed in the PSM (Fig. 4G). The anterior somite marker EphA4 was expressed in two domains in wild-type embryos - the anterior half of somite I (Fig. 4H, arrowhead) and the anterior region of the PSM (Fig. $4 \mathrm{H}$, arrow). In Hes7-null embryos, EphA4 expression in somite I became almost undetectable, whereas the expression in the PSM was not significantly affected (Fig. 4H). These results indicate that the A-P polarity of somites was severely disorganized in Hes7-null mice.

Because Hes 7 is a putative downstream effector of the Notch pathway, we next analyzed the expression of Notch receptors and ligands. In the wild type, Notch1 was expressed in the PSM with a sharp anterior border between somites I and -I, whereas in the Hes7-mutant embryo, the expression in the PSM was observed but the anterior border was diffuse (Fig. 5A). Notch2 was expressed in the anterior half of the newly formed somite and the whole region of the next presumptive somite (somite -I) of the wild type, whereas in Hes7-null embryos, only a broader expression domain was observed in the anterior PSM (Fig. 5B). The Notch ligand genes D111 and D113 were expressed in the PSM with a sharp anterior border in the wild-type embryo (Fig. 5C,D). These genes were expressed in the mutant PSM, although the anterior border was more diffuse and Dl11 expression in the posterior half of each somite became almost undetectable (Fig. 5C,D). These results indicate that, although expression of the receptors and ligands of Notch signaling became diffuse at the anterior border of the PSM and undetectable in somites, the overall expression level in the PSM was not affected by loss of Hes7.

\section{Disruption of oscillating gene expression in Hes7-mutant mice}

We next examined expression of the oscillator genes Hes1 and Hey2 (Jouve et al. 2000; Leimeister et al. 2000). In the wild type, both Hes1 and Hey2 were expressed in the PSM and the posterior half of somites (Fig. 5E,F). In the Hes7-null embryo, although Hes1 expression remained in somite -I (Fig. 5E, bracket), it became undetectable in the posterior PSM and somites (Fig. 5E). In addition, Hey2 expression was also undetectable in the PSM of Hes7-null embryos (Fig. 5F). Therefore, Hes 7 is essential for Hes1 and Hey2 expression in the PSM. We next examined another oscillator gene, $L f n g$. In the wildtype and heterozygous embryos, Lfng displayed various expression patterns categorized into three stages (Fig. 5G). In contrast, we did not observe such variability of Lfng expression in Hes7-null embryos (Fig. 5H). Strikingly, all Hes7-null embryos displayed the same expression pattern ( $n=8$ at E8.5 and $n=9$ at E9.5). The expression was observed continuously throughout the PSM with the highest in the anterior PSM, probably at somite -II (Fig. 5H). In addition, the same abnormal expression pattern was observed at both E8.5 and E9.5 (Fig. 5H), indicating that the defect of $L f n g$ expression is not progressing but rather constant. This expression pattern of Lfng was never observed at any stages in the wild-type and heterozygous embryos (Fig. 5G). These results strongly indicate that the oscillating expression of Hes1, 


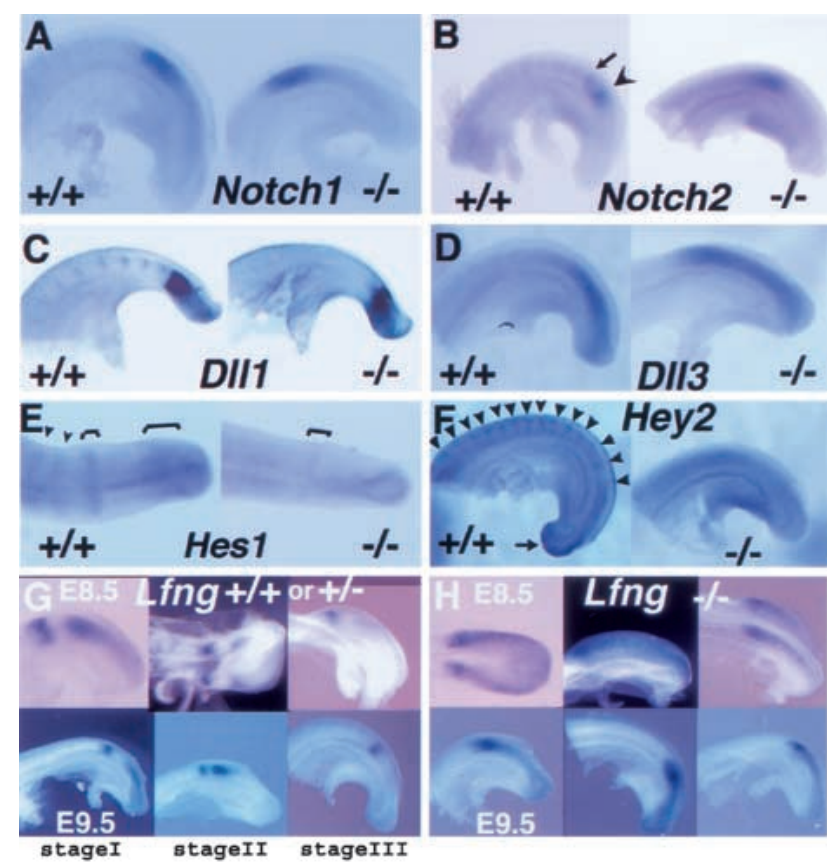

Figure 5. Whole-mount in situ hybridization for Notch signaling molecules. $(A)$ In the Hes7-null embryo, the expression is not significantly affected in the PSM, but the anterior border becomes diffuse. $(B)$ In the wild type, Notch2 is expressed in somites I (arrow) and -I (arrowhead). In the Hes7-null embryo, only a broader expression is observed in the anterior PSM. (C) In the wild type, DIl1 is expressed in the PSM and the posterior half of each somite. In the Hes7-null embryo, it is expressed in the PSM, but the anterior border is diffuse and the somite expression is undetectable. $(D)$ DIl3 expression in the PSM is not significantly affected but the anterior border is diffuse in Hes7null embryos. (E) In the wild type, Hes1 is expressed in the PSM (brackets) and the posterior half of somites (arrowheads). In the Hes7-null embryo, the expression is observed in somite -I (bracket) but undetectable in other regions $(n=5)$. ( $F)$ In the wild type, Hey2 is expressed in the PSM (arrow) and the posterior half of somites (arrowheads). In the Hes7-null embryo, the expression is almost undetectable $(n=4)$. (G) In the wild type, Lfng displays various expression patterns at E8.5 (stage I, 9; stage II, 16; stage III, 12 out of 37 embryos) and E9.5 (stage I, 6; stage II, 9; stage III, 10 out of 25 embryos). (H) In contrast, all Hes7-null embryos (E8.5, $n=8$; E9.5, $n=9$ ) exhibit the same expression pattern-Lfng is expressed continuously throughout the mutant PSM.

Hey2, and Lfng in the PSM was all disrupted in Hes7null mice.

Here, we showed that mutation for a new member of Hes family, Hes7, leads to severe defects of somite segmentation. Strikingly, in Hes7-null embryos, Lfng expression was expressed continuously throughout the PSM, and therefore the cyclic expression of Lfng was disrupted. Because Hes7 is a transcriptional repressor (Bessho et al. 2001), it may down-regulate Lfng expression and generate a Lfng-negative region in the PSM during oscillation. Our data do not, however, exclude the possibility that $L$ fng expression still oscillates in individual cells in Hes7-null embryos. Interestingly, the phenotypes of Hes7-deficient mice are very similar to those of Lfng-null mice (Evrard et al. 1998; Zhang and Gridley
1998). Therefore, both null mutation and continuous expression of $L$ fng result in somite defects, suggesting that the oscillating expression is essential for coordinated somite segmentation.

Although Hes7 seems to repress Lfng and its own expression, both Hes7 and Lfng mRNAs are generated in the same phase during oscillation in the PSM, suggesting that Hes7 protein is unlikely to function in $L$ fng/Hes 7 mRNA $^{+}$domains. Previous analysis of the immediateearly gene c-fos suggests that the peak of the protein level is $~ 30$ min later than that of the mRNA level (Müller et al. 1984). Therefore, the synthesis of Hes7 protein is likely to be delayed compared with the production of Hes 7 mRNA. We speculate that the Hes7 protein may function in the regions just caudal to the peak of $L f n g$ and Hes $7 \mathrm{mRNA}^{+}$domain, although further studies are required to determine the position of the peak of Hes7 protein level.

\section{Materials and methods}

\section{Generation of Hes7 mutant mice}

Targeting vector was constructed by replacing most of the Hes7-coding region to IRES-LacZ and PGK-neo (Fig. 2A). ES cell lines with Hes7 mutation were identified by Southern blot analysis using $5^{\prime}$ and $3^{\prime}$ probes (Fig. 2A,B). Chimeric mice were generated and bred with ICR mice, as described previously (Tomita et al. 2000).

Explant culture of mouse embryos

The caudal part of E9.5 mouse embryo was bisected along the midline in fetal bovine serum. The explants were cultured on polycarbonate filters (Millipore) in DMEM (GIBCO) supplemented with $20 \%$ fetal bovine serum, as described previously (Palmeirim et al. 1997).

In situ hybridization and lacZ staining

Whole-mount in situ hybridization of mouse embryos was performed as described previously (Bessho et al. 2001). Notch1, Notch2, and Dll1 cDNAs were kindly provided by Dr. Gerry Weinmaster. Other probes were obtained by RT-PCR and the following regions were used: Dll3, nucleotide residues 612-1341; Lfng, 17-1382; Mesp2, 1-1697; Uncx4.1, -141680; Paraxis, 106-837; pMesogenin, 2-547; EphA4, 1550-2960; EphrinB2, -1-2143; Hes1, -178-1213; and Hey2, -135-1020. To assess the expression of the $L a c Z$ gene, embryos were fixed in $0.2 \%$ glutaraldehyde and then stained in $5 \mathrm{mM}$ potassium ferricyanide, $5 \mathrm{mM}$ potassium ferrocyanide, $2 \mathrm{mM} \mathrm{MgCl} 2$ and $1 \mathrm{mg} / \mathrm{mL}$ 5-bromo-4-chloro-3-indolyl- $\beta$-Dgalactoside.

\section{Skeletal preparation}

Cartilages and bones of newborn mice were stained with alcian blue and alizanin red after fixation in 95\% ethanol as described by Inouye (1976).

\section{Acknowledgments}

We thank Dr. Olivier Pourquié for critically reading the manuscript and important discussion; Drs. Yoichi Shinkai and Hiromi Hirata for technical help; and Dr. Gerry Weinmaster for reagents. This work was supported by research grants from the Ministry of Education, Culture, Sports, Science and Technology of Japan and the Japan Society for the Promotion of Science.

The publication costs of this article were defrayed in part by payment of page charges. This article must therefore be hereby marked "advertisement" in accordance with 18 USC section 1734 solely to indicate this fact.

\section{References}

Aulehla, A. and Johnson, R.L. 1999. Dynamic expression of $L f n g$ suggests a link between notch signaling and an autonomous cellular oscillator driving somite segmentation. Dev. Biol. 207: 49-61.

Bessho, Y., Miyoshi, G., Sakata, R., and Kageyama, R. 2001. Hes7: A 
bHLH-type repressor gene regulated by Notch and expressed in the presomitic mesoderm. Genes Cells 6: 175-185.

Burgess, R., Rawls, A., Brown, D., Bradley, A., and Olson, E.N. 1996 Requirement of the paraxis gene for somite formation and musculoskeletal patterning. Nature 384: 570-573.

Conlon, R.A., Reaume, A.G., and Rossant, J. 1995. Notch1 is required for the coordinate segmentation of somites. Development 121: 15331545.

Cooke, J. 1998. A gene that resuscitates a theory-Somitogenesis and a molecular oscillator. Trends Genet. 14: 85-88.

Dale, K.J. and Pourquié, O. 2000. A clock-work somite. BioEssays 22: $72-83$.

del Barco Barrantes, I., Elia, A.J., Wünsch, K., Hrabe de Andelis, M., Mak, T.W., Rossant, J., Conlon, R.A., Gossler, A., and de la Pompa, J.L. 1999. Interaction between Notch signaling and Lunatic fringe during somite boundary formation in the mouse. Curr. Biol. 9: 470-480.

Donoviel, D.B., Hadjantonakis, A.-K., Ikeda, M., Zheng, H., Hyslop, P.S.G., and Bernstein, A. 1999. Mice lacking both presenilin genes exhibit embryonic patterning defects. Genes \& Dev. 13: 2801-2810.

Evrard, Y.A., Lun, Y., Aulehla, A., Gan, L., and Johnson, R.L. 1998. lunatic fringe is an essential mediator of somite segmentation and patterning. Nature 394: 377-381.

Forsberg, H., Crozet, F., and Brown, N.A. 1998. Waves of mouse Lunatic fringe expression, in four-hour cycles at two-hour intervals, precede somite boundary formation. Curr. Biol. 8: 1027-1030.

Holley, S.A., Geisler, R., and Nüsslein-Volhard, C. 2000. Control of her1 expression during zebrafish somitogenesis by a Delta-dependent oscillator and an independent wave-front activity. Genes \& Dev. 14: $1678-1690$.

Hrabe de Angelis, M., McIntyre II, J., and Gossler, A. 1997. Maintenance of somite borders in mice requires the Delta homologue Dll1. Nature 386: 717-721

Huppert, S.S., Le, A., Schroeter, E.H., Mumm, J.S., Saxena, M.T., Milner, L.A., and Kopan, R. 2000. Embryonic lethality in mice homozygous for a processing-deficient allele of Notch1. Nature 405: 966-970

Inouye, M. 1976. Differential staining of cartilage and bone in fetal mouse skeleton by alcian blue and alizarin red S. Cong. Anom. 16: 171-173.

Ishibashi, M., Ang, S.-L., Shiota, K., Nakanishi, S., Kageyama, R., and Guillemot, F. 1995. Targeted disruption of mammalian hairy and Enhancer of split homolog-1 (HES-1) leads to up-regulation of neural helix-loop-helix factors, premature neurogenesis, and severe neural tube defects. Genes \& Dev. 9: 3136-3148.

Jiang, Y.J., Aerne, B.L., Smithers, L., Haddon, C., Ish-Horowicz, D., and Lewis, J. 2000. Notch signalling and the synchronization of the somite segmentation clock. Nature 408: 475-479.

Jouve, C., Palmeirim, I., Henrique, D., Beckers, J., Gossler, A., IshHorowicz, D., and Pourquie, O. 2000. Notch signalling is required for cyclic expression of the hairy-like gene HES1 in the presomitic mesoderm. Development 127: 1421-1429.

Koizumi, K., Nakajima, M., Yuasa, S., Saga, Y., Sakai, T., Kuriyama, T., Shirasawa, T., and Koseki, H. 2001. The role of presenilin 1 during somite segmentation. Development 128: 1391-1402.

Kusumi, K., Sun, E.S., Kerrebrock, A.W., Bronson, R.T., Chi, D.-C., Bulotsky, M.S., Spencer, J.B., Birren, B.W., Frankel, W.N., and Lander, E.S. 1998. The mouse pudgy mutation disrupts Delta homologue Dll3 and initiation of early somite boundaries. Nat. Genet. 19: 274 278.

Leimeister, C., Dale, K., Fischer, A., Klamt, B., Hrabe de Angelis, M., Radtke, F., McGrew, M.J., Pourquie, O., and Gessler, M. 2000. Oscillating expression of c-Hey2 in the presomitic mesoderm suggests that the segmentation clock may use combinatorial signaling through multiple interacting bHLH factors. Dev. Biol. 227: 91-103.

Mansouri, A., Voss, A.K., Thomas, T., Yokota, Y., and Gruss, P. 2000. Uncx4.1 is required for the formation of the pedicles and proximal ribs and acts upstream of Pax9. Development 127: 2251-2258.

McGrew, M.J., Dale, J.K., Fraboulet, S., and Pourquié, O. 1998. The lunatic fringe gene is a target of the molecular clock linked to somite segmentation in avian embryo. Curr. Biol. 8: 979-982.

Müller, R., Bravo, R., Burckhardt, J., and Curran, T. 1984. Induction of c- $f o s$ gene and protein by growth factors precedes activation of c-myc. Nature 312: 716-720.

Oka, C., Nakano, T., Wakeham, A., de la Pompa, J.L., Mori, C., Sakai, T.,
Okazaki, S., Kawaichi, M., Shiota, K., Mak, T.W., and Honjo, T. 1995 Disruption of the mouse RBP-J kappa gene results in early embryonic death. Development 121: 3291-3301.

Palmeirim, I., Henrique, D., Ish-Horowicz, D., and Pourquie, O. 1997. Avian hairy gene expression identifies a molecular clock linked to vertebrate segmentation and somitogenesis. Cell 91: 639-648.

Saga, Y., Hata, N., Koseki, H., and Taketo, M.M. 1997. Mesp2: A novel mouse gene expressed in the presegmented mesoderm and essential for segmentation initiation. Genes \& Dev. 11: 1827-1839.

Sawada, A., Fritz, A., Jiang, Y.-J., Yamamoto, A., Yamasu, K., Kuroiwa, A., Saga, Y., and Takeda, H. 2000. Zebrafish Mesp family genes, mesp- $a$ and mesp- $b$ are segmentally expressed in the presomitic mesoderm, and Mesp-b confers the anterior identity to the developing somites. Development 127: 1691-1702.

Stern, C.D. and Keynes, R.J. 1987. Interactions between somite cells: The formation and maintenance of segment boundaries in the chick embryo. Development 99: 261-272.

Swiatek, P.J., Lindsell, C.E., Franco del Amo, F., Weinmaster, G., and Gridley, T. 1994. Notch1 is essential for postimplantation develop ment in mice. Genes \& Dev. 8: 707-719.

Tomita, K., Moriyoshi, K., Nakanishi, S., Guillemot, F., and Kageyama, R. 2000. Mammalian achaete-scute and atonal homologs regulate neuronal versus glial fate determination in the central nervous system. EMBO J. 19: 5460-5472.

Wong, P.C., Zheng, H., Chen, H., Becher, M.W., Sirinathsinghii, D.J.S. Trumbauer, M.E., Chen, H.Y., Price, D.L., Van der Ploeg, L.H.T., and Sisodia, S.S. 1997. Presenilin 1 is required for Notch1 and Dll1 expression in the paraxial mesoderm. Nature 387: 288-292.

Yoon, J.K. and Wold, B. 2000. The bHLH regulator pMesogenin1 is required for maturation and segmentation of paraxial mesoderm. Genes \& Dev. 14: 3204-3214.

Zhang, N. and Gridley, T. 1998. Defects in somite formation in lunatic fringe-deficient mice. Nature 394: 374-377. 


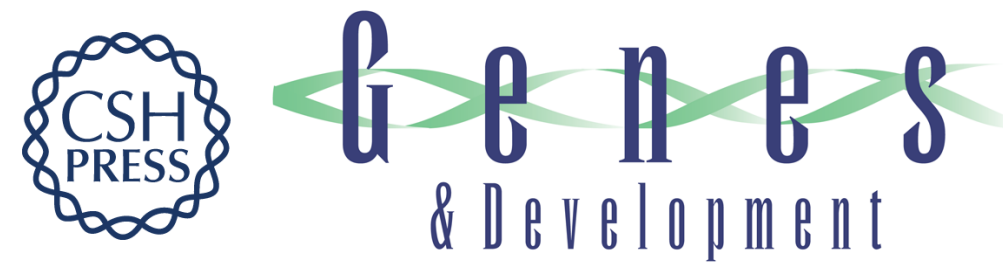

\section{Dynamic expression and essential functions of Hes7 in somite segmentation}

Yasumasa Bessho, Ryoichi Sakata, Suguru Komatsu, et al.

Genes Dev. 2001, 15:

Access the most recent version at doi:10.1101/gad.930601

References

This article cites 33 articles, 14 of which can be accessed free at: http://genesdev.cshlp.org/content/15/20/2642.full.html\#ref-list-1

\section{License}

Email Alerting

Receive free email alerts when new articles cite this article - sign up in the box at the top Service right corner of the article or click here.

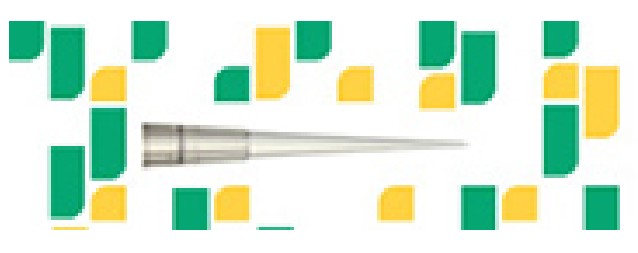

Focused on your science. 